\title{
Caracterización por microscopía electrónica de barrido del recubrimiento no electrolítico de níquel (electroless nickel) sobre piezas de hierro boronizado
}

\section{Characterization by Scanning Electron Microscopy of the "Electroless Nickel" coating on difusion-borided iron parts}

\author{
Nelson Gomes ${ }^{1}$, Amnon Vadasz ${ }^{2}$, Joaquín Brito ${ }^{3}$, Myloa Morgado-Vargas ${ }^{4}$, Susana Pinto-Castilla ${ }^{5}$ \\ ${ }^{1}$ Unidad de Control y Gestión de Desechos Químicos, Centro de Química, Instituto Venezolano de Investigaciones Científicas \\ (IVIC), Venezuela. Correo electrónico: bureta2001@ @otmail.com \\ ${ }^{2}$ C. A. Tecnología Aplicada Venezolana, Venezuela. Correo electrónico: amnon.vadasz@ gmail.com \\ ${ }^{3}$ Yachay Tech University, 100119 - Urcuqui, Ecuador. Correo electrónico: jbrito@yachaytech.edu.ec \\ ${ }^{4}$ Unidad de Microscopía Electrónica, Centro de Química, Instituto Venezolano de Investigaciones Científicas (IVIC), Venezuela. \\ Correo electrónico: myloamorgado@gmail.com \\ ${ }^{5}$ Laboratorio Fisicoquímica de Superficie, Centro de Química, Instituto Venezolano de Investigaciones Científicas (IVIC), \\ Venezuela. Correo electrónico: cspc99@gmail.com
}

Recibido: 28 febrero, 2018. Aceptado: 6 de mayo, 2018. Versión final: 22 junio, 2018.

\begin{abstract}
Resumen
El presente estudio es un primer acercamiento para determinar la factibilidad en el uso del recubrimiento denominado electroless nickel, como una opción para el reforzamiento anticorrosivo de piezas boronizadas, las cuales pueden presentar microgrietas durante su uso normal. Para este estudio, se evaluó si hay difusión del recubrimiento en el compuesto intermetálico. Se emplearon muestras industriales de tubería de acero al carbono J55 boronizado, según proceso EndurAlloyMR (por Endurance Technologies Inc., Calgary, Canadá). Las probetas fueron limpiadas y decapadas para luego ser recubiertas y tratadas térmicamente. La caracterización morfológica se llevó a cabo por microscopía electrónica de barrido (MEB). La distribución elemental en la zona de contacto entre el recubrimiento y el compuesto intermetálico se determinó empleando la técnica EDX-Mapping, y la zona de difusión se determinó empleando la técnica EDX-LineScan. Los resultados obtenidos en el análisis elemental permitieron comprobar la existencia de una zona de difusión entre el recubrimiento y el compuesto intermetálico de aproximadamente $5 \mu \mathrm{m}$ de espesor, lo que se traduce en una excelente adherencia, al tiempo que aumenta la probabilidad de cubrir las microgrietas y preserva la protección anticorrosiva de piezas boronizadas.
\end{abstract}

Palabras clave: boronizado; compuesto intermetálico; difusión; recubrimiento no electrolítico.

\begin{abstract}
The present study is a first approach to determine the feasibility of using the "Electroless Nickel" coating as an option for the anticorrosive reinforcement of borided parts, which may present micro-cracks during normal use. For this study, is the presence of diffusion of the coating in the intermetallic compound was evaluated. We used industrial samples of borided carbon steel J55 pipe according to EndurAlloyMR process (by Endurance Technologies Inc., Calgary, Canada). The test pieces were cleaned, pickled, coated and heat-treated. The morphological characterization was carried out by Scanning Electron Microscopy (SEM). The elemental distribution in the contact zone between the coating and the intermetallic compound was determined using the EDX - Mapping technique and the diffusion area was determined using the EDX - LineScan technique. The results obtained in the elemental analysis allowed to verify the existence of a diffusion zone between the coating and the intermetallic compound of approximately $5-\mu \mathrm{m}$ thickness,
\end{abstract}

ISSN impreso: 1657 - 4583, ISSN en línea: 2145 - 8456, CC BY-ND 4.0 (c) (i)

Como citar: N. Gomes, A. Vadasz, J. Brito, M. Morgado-Vargas, S. Pinto-Castilla, "Caracterización por microscopía electrónica de barrido del recubrimiento no electrolítico de níquel (electroless nickel) sobre piezas de hierro boronizado,” Rev. UIS Ing., vol. 18, no. 1, pp. 127-140, 2019. doi: 10.18273/revuin.v18n1-2019011 
which translates into an excellent adhesion property, increasing the probability of covering the micro-cracks and preserving the anticorrosive protection of borided parts.

Keywords: electroless coating; boronizing; intermetallic compound; diffusion.

\section{Introducción}

\subsection{Recubrimiento de níquel fosforado}

Los fosfuros de níquel son usados en el campo de la metalurgia en un tipo de recubrimiento metálico denominado 'níquel fosforado', que se aplica sobre diversos tipos de metales y aleaciones. Este tipo de recubrimiento se obtiene mediante la técnica denominada 'deposición química no electrolítica', de modo que se produce una mezcla de níquel y fósforo en diversas proporciones, donde el contenido de fósforo juega un papel fundamental en las propiedades finales de la capa depositada, entre las cuales se puede mencionar la protección anticorrosiva de las piezas, resistencia al desgaste y dureza para diversas aplicaciones [1].

La técnica de deposición química no electrolítica es un método autocatalítico, que consiste en la reducción de iones metálicos en solución y la deposición de una película de espesor específico, todo esto en ausencia de una fuente externa de corriente eléctrica. La película resultante es un codepósito formado por el metal y un elemento no metálico proveniente del agente reductor empleado. Los recubrimientos de níquel fosforado se forman a partir de esta técnica, en la cual se emplea como fuente de los iones metálicos una sal de níquel que puede ser $\mathrm{NiCl}_{2} \mathrm{O} \mathrm{NiSO}_{4}$. Como agente reductor y fuente de fósforo se usa el hipofosfito de sodio [2-11].

La técnica de recubrimiento fue reportada por primera vez por Brenner y Riddell [2], quienes expusieron un método estable de recubrimiento de metales que no requería el uso de electricidad, el cual se basaba en sumergir la pieza metálica en un baño de $\mathrm{NiCl}_{2}$ y $\mathrm{NaH}_{2} \mathrm{PO}_{2}$ a un intervalo de $\mathrm{pH}$ de 8-9 y temperaturas de $90{ }^{\circ} \mathrm{C}$. West [3] estudió los baños de recubrimiento y determinó que parámetros como el $\mathrm{pH}$ y la temperatura influyen en la velocidad de deposición y la calidad del recubrimiento. El mismo autor [4] también reportó un estudio del recubrimiento en materiales no ferrosos, del que se obtuvieron capas de níquel fosforado en metales como aluminio, latón, cobre y titanio, cuando hasta ese momento solo se había reportado en materiales ferrosos.

El recubrimiento de níquel fosforado se aplica industrialmente a piezas nuevas o usadas [1] para

- Ofrecer una protección contra la corrosión, el desgaste y la erosión/corrosión de la superficie metálica.
- Reemplazar directamente al cromo duro o al termorrociado, cuando exista la posibilidad o haya experiencia previa de que la pieza pueda desconcharse.

Para piezas usadas, el recubrimiento se aplica para

- Restauración de medidas (sea por uso o error de mecanizado).

- Corrección de daños ocasionados por golpes o desprendimientos de capa de áreas previamente revestidas.

Dependiendo de la aplicación específica, el espesor de capa varía entre 5 y 150 micrómetros. Las propiedades fisicoquímicas del recubrimiento varían en función del contenido de fósforo en la solución sólida y del tratamiento térmico al que se somete la capa posteriormente. La composición del baño químico puede influir en la aparición de porosidad en la capa, así como también en la resistencia a la corrosión.

El níquel fosforado se puede depositar a partir de baños químicos alcalinos o ácidos. Los baños alcalinos se utilizan para aplicaciones a baja temperatura $\left(<60^{\circ} \mathrm{C}\right)$, pero, debido a su baja velocidad de deposición, no son prácticos industrialmente. Los baños ácidos, por el contrario, permiten usar una mayor temperatura, con lo que se puede lograr una mayor velocidad de deposición y una capa menos porosa y con mayor resistencia a la corrosión, al desgaste y a la erosión/corrosión. Es por ello que estos últimos son los que se utilizan para la protección y reconstrucción de componentes críticos (con énfasis en partes de equipos) o para la protección de piezas que estén sometidas a ambientes agresivos [12].

Dependiendo del valor del $\mathrm{pH}$ en el baño de recubrimiento, es posible realizar la deposición de relaciones porcentuales específicas de níquel y fósforo en el mismo baño [9]. En la medida en que la alcalinidad de los baños aumenta, el contenido de níquel en el depósito se incrementa mientras que el de fósforo disminuye.

En cuanto a las superficies de trabajo, el recubrimiento se puede aplicar sobre distintos tipos de metales: aceros carbono, aceros inoxidables, aluminio, bronce, cobre, titanio, sobre rellenos de soldadura y algunas otras aleaciones, previa activación adecuada. Igualmente se pueden recubrir sustratos no metálicos como diversos polímeros (ABS, Nylon, PVC) y vidrio [12]. 
La capa de níquel-fósforo, una vez depositada sobre la pieza, puede ser sometida a un tratamiento térmico a una temperatura determinada (generalmente entre 200 y 400 ${ }^{\circ} \mathrm{C}$ ), en el que se generan diferentes fases cristalinas de fosfuros de níquel, entre las que se pueden destacar NiP, $\mathrm{Ni}_{2} \mathrm{P}, \mathrm{Ni}_{3} \mathrm{P}, \mathrm{Ni}_{12} \mathrm{P}_{5}$ y $\mathrm{Ni}_{5} \mathrm{P}_{2}$ [6-9], además de níquel cristalin. ${ }^{\circ}$ Estos tratamientos térmicos se realizan en las piezas recubiertas cuando es necesario aumentar la dureza y la resistencia al desgaste del recubrimiento.

Graham y colaboradores [5] publicaron uno de los primeros estudios de difracción de rayos-X (DRX) sobre recubrimientos de níquel fosforado. Plantearon que estos son «soluciones sólidas supersaturadas de fósforo disuelto en una matriz microcristalina de níquel». Estudiaron la influencia de la variación del contenido de fósforo en el recubrimiento, así como del tratamiento térmico, sobre la estructura y propiedades mecánicas del recubrimiento. Generaron capas entre 4,6 y $9,4 \%$ en peso de fósforo y realizaron tratamientos térmicos en argón, a un intervalo de temperatura de entre 200 y $750{ }^{\circ} \mathrm{C}$. Las capas obtenidas por encima de $\operatorname{los} 400{ }^{\circ} \mathrm{C}$ presentaron pérdida de dureza y adherencia al sustrato. Las fases detectadas en los análisis por difracción de electrones fueron las de $\mathrm{Ni}_{3} \mathrm{P}$ y Ni.

Apachitei y Duszczyk [7] estudiaron los recubrimientos de níquel fosforado realizados con baños ácidos sobre aluminio, en codeposición con carburo de silicio. Para ello realizaron la deposición en varias piezas a diferentes relaciones de fósforo en un intervalo de 2,5 a 10,2 \% en peso. Los análisis por DRX de las muestras, previo al tratamiento térmico (véase figura 1), mostraron una tendencia a obtener una mayor cristalinidad del níquel en la medida en que disminuye el contenido de fósforo en la capa.

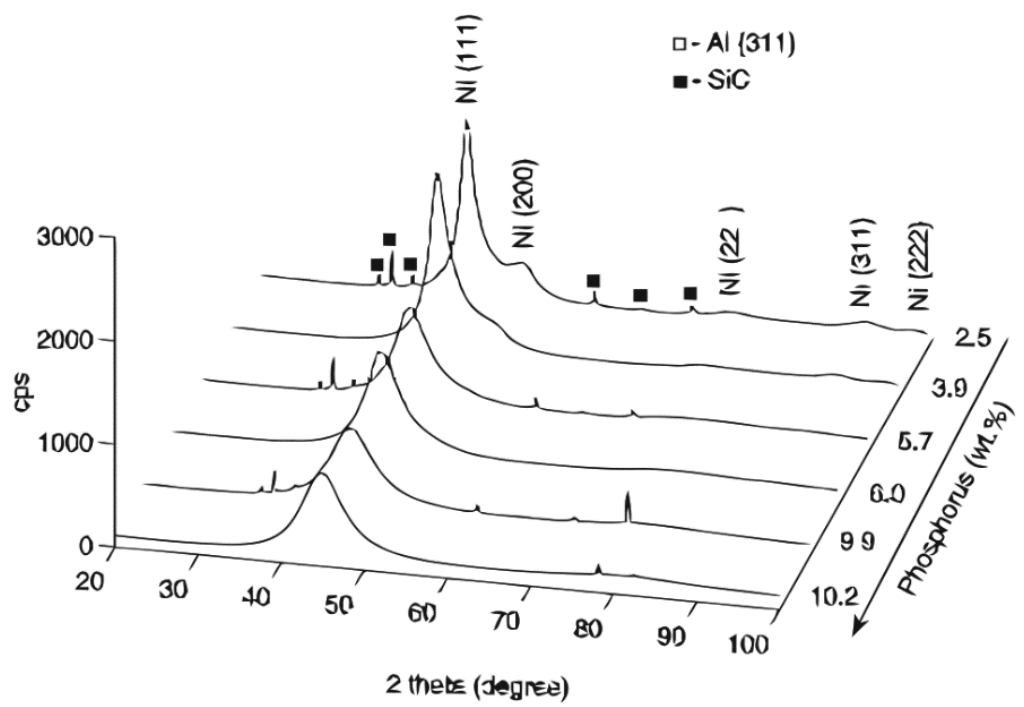

Figura 1. Patrones de difracción de recubrimientos de níquel fosforado codepositado con carburo de silicio, en el intervalo de concentraciones de 2,5\% a 10,2 \% de fósforo. Fuente: Apachitei y Duszczyk [7].

Keong y colaboradores [10] realizaron un estudio detallado sobre la cinética de cristalización y la transformación de fases del recubrimiento para un intervalo de temperaturas de entre 300 y $800{ }^{\circ} \mathrm{C}$ a $20{ }^{\circ} \mathrm{C} / \mathrm{min}$ en aire, en muestras con concentraciones entre Esta estructura nodular ha sido ampliamente reportada por diversos autores en la bibliografía, entre los cuales destacan Fan y colaboradores [13], quienes realizaron recubrimientos de fósforo medio sobre aleaciones de magnesio. Estos autores reportaron una estructura nodular de tipo coliflor (véase figura 4) con una dispersión de poros en las intersecciones entre los nódulos 10 y $16 \%$ en peso de fósforo (véase figura 2).
Las fases predominantes a bajas temperaturas fueron $\mathrm{Ni}$, $\mathrm{Ni}_{2} \mathrm{P}, \mathrm{Ni}_{12} \mathrm{P}_{5}$ y $\mathrm{Ni}_{3} \mathrm{P}$, mientras que a altas temperaturas permanecieron las fases $\mathrm{Ni}$ y $\mathrm{Ni}_{3} \mathrm{P}$, con la aparición de óxido de níquel.

En cuanto a su morfología y topografía, los recubrimientos presentan una superficie nodular (véase figura 3), denominada "tipo coliflor" por algunos autores, debido a la forma y la distribución ordenada de los nódulos (semiesferas de diversos diámetros) que la componen. 


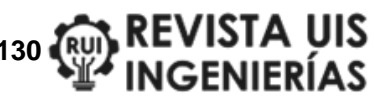

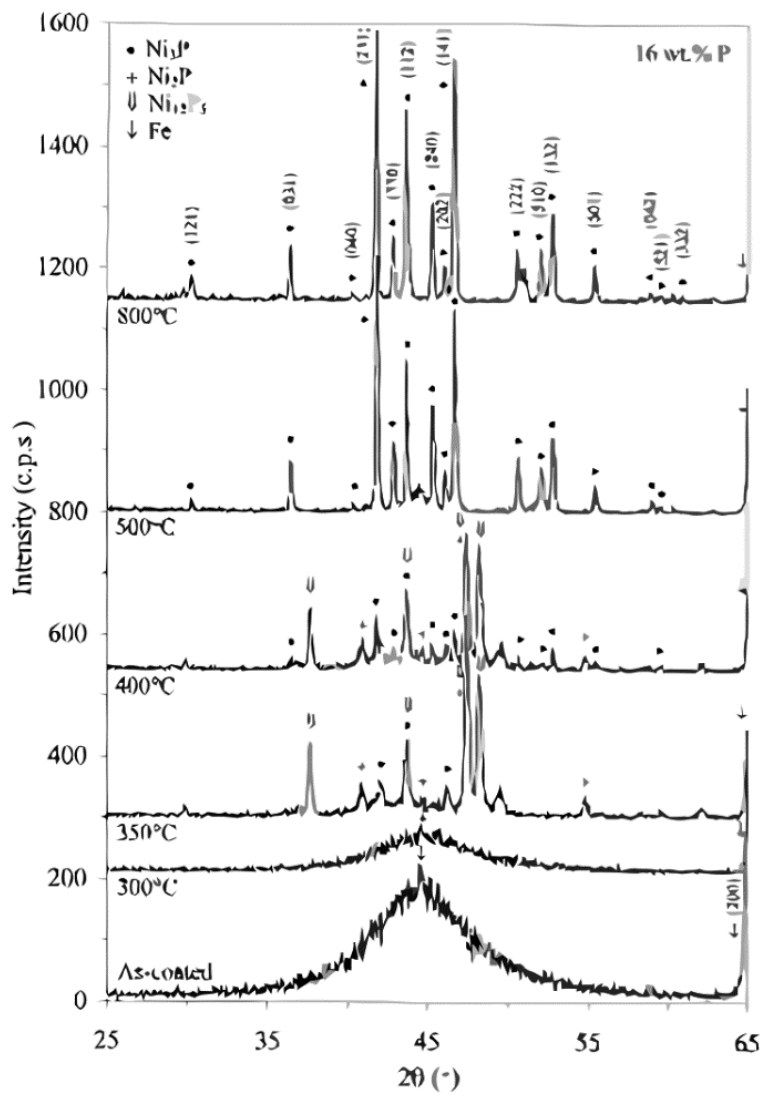

N. Gomes, A. Vadasz, J. Brito, M. Morgado, S. Pinto-Castilla

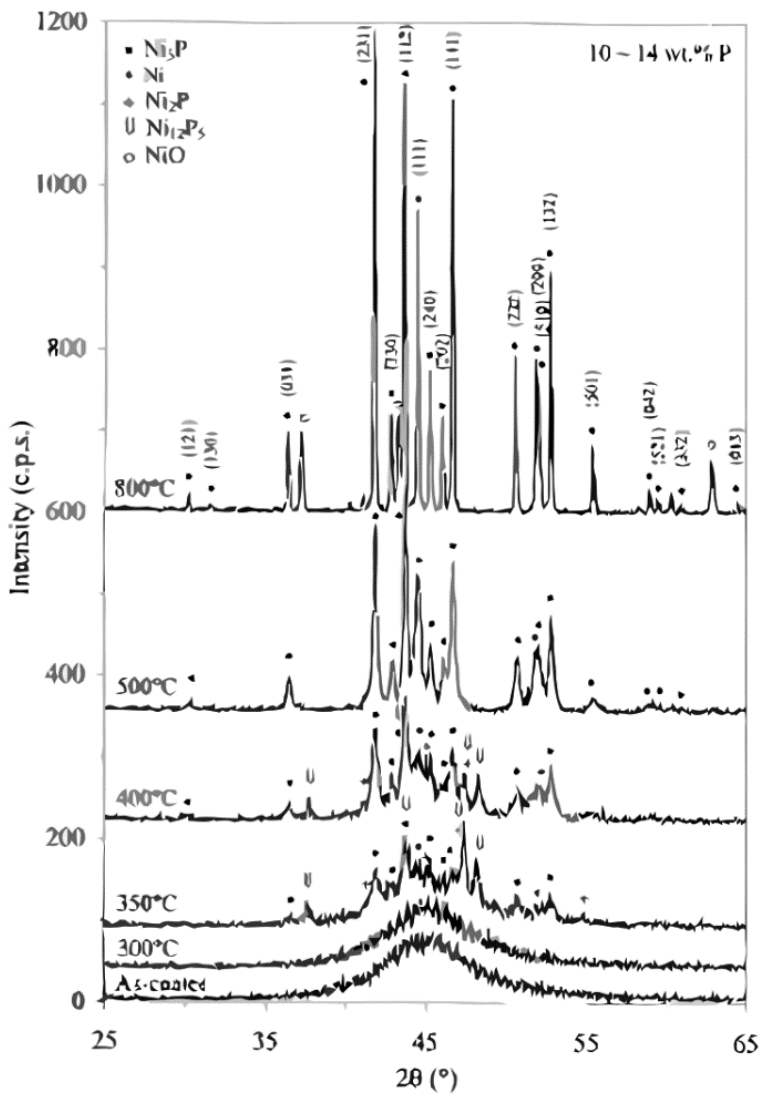

Figura 2. Patrones de difracción de recubrimientos de níquel fosforado con distintos contenidos de fósforo (P), antes y después del tratamiento térmico a diferentes temperaturas: a) $16 \%$ de P; b) 10-14 \% de P. Fuente: Keong et al.

[10].

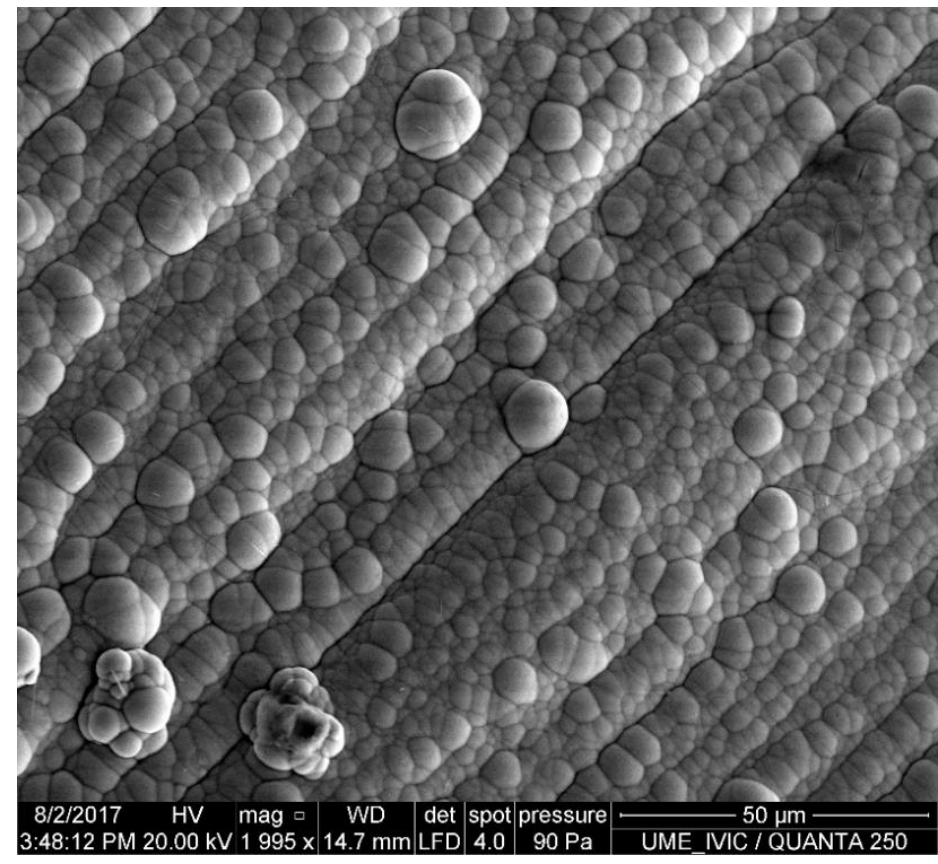

Figura 3. Morfología nodular típica del recubrimiento de níquel fosforado. Fuente: elaboración propia. 


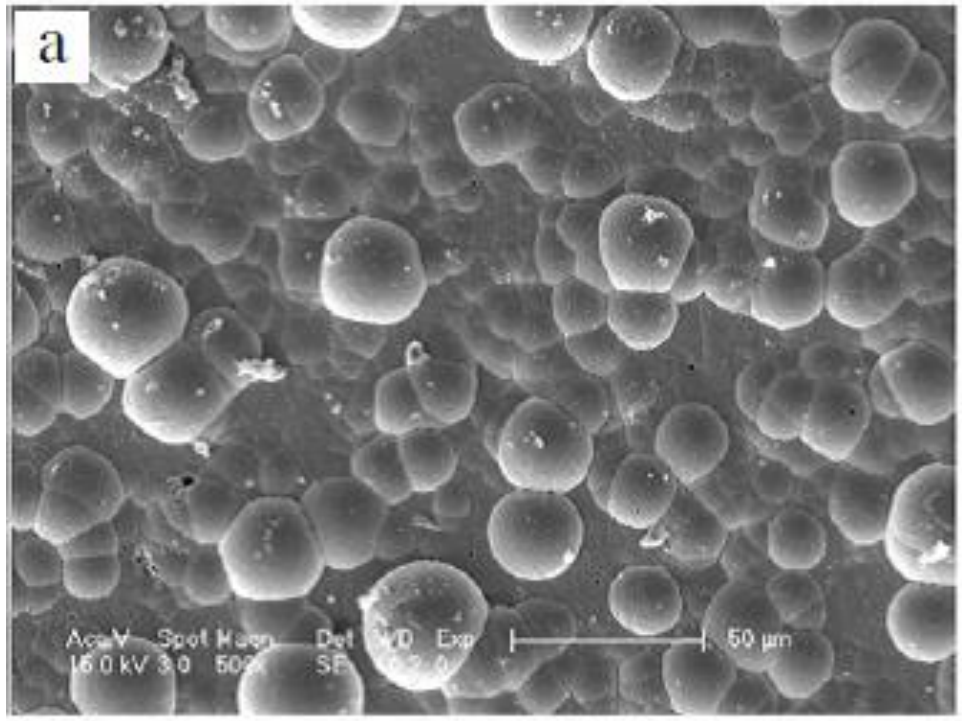

Figura 4. Micrografía de recubrimiento de níquel fosforado. Fuente: Fan et al [13].

Seifzadeh y colaboradores [14] realizaron un estudio de recubrimiento de fósforo medio sobre una aleación de magnesio AM60B. Los autores reportan la formación del arreglo típico de coliflor e indican que es característico de los recubrimientos amorfos (sin tratamiento térmico posterior al recubrimiento). Baskaran y colaboradores [15] estudiaron la influencia de los agentes estabilizantes en los baños de deposición química, empleando como sustrato láminas de cobre grado ETP. Realizaron ensayos a un $\mathrm{pH}$ de 4,5 y diversas composiciones de baño, y obtuvieron la misma estructura de coliflor ya citada (véase figura 5). En este caso, los autores plantean que la rápida formación de núcleos se debe a la alta velocidad de deposición que poseen los baños realizados a un $\mathrm{pH}$ entre 4 y 7. Estas altas velocidades favorecen el crecimiento nodular preferencialmente en la dirección $\mathrm{Z}$, en lugar de las direcciones X y Y, debido al constante y rápido suministro de átomos de níquel y fósforo provenientes de los precursores en el baño.

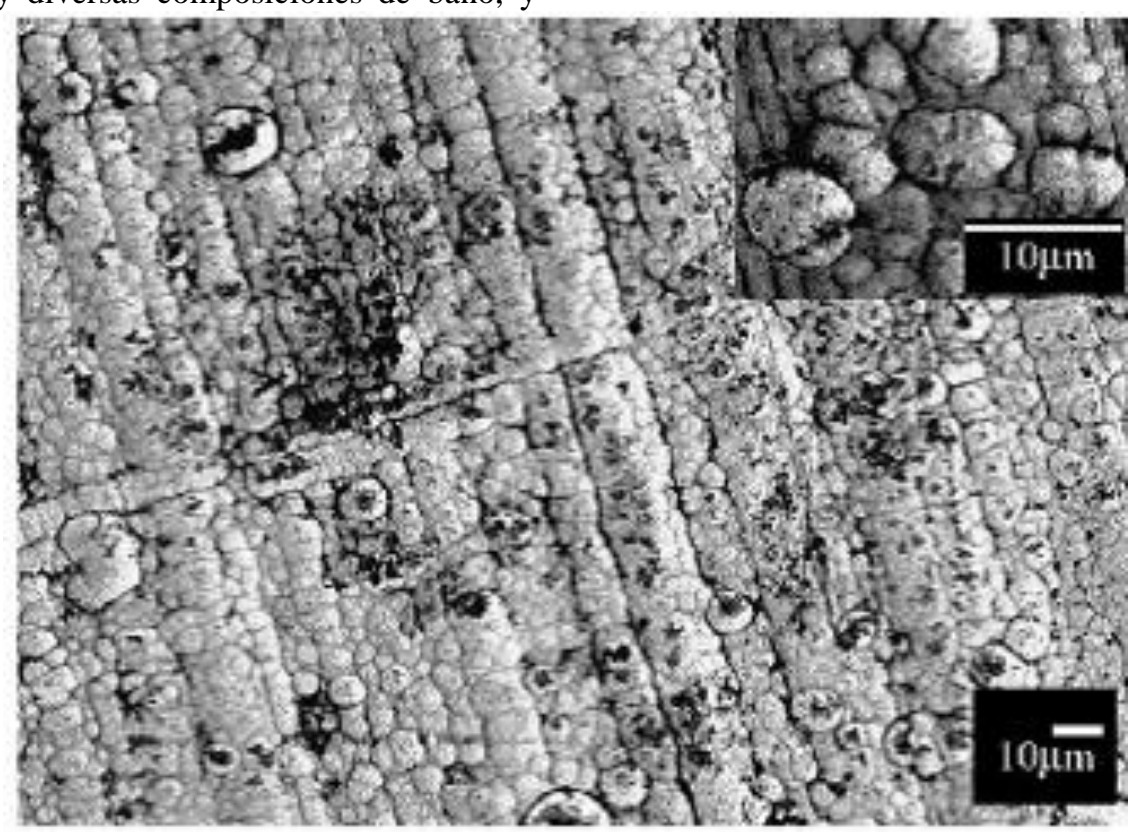

Figura 5. Micrografía de recubrimiento de níquel fosforado. Fuente: Baskaran et al [15]. 


\subsection{Difusión de boro o boronizado}

El borizado o boronizado es una técnica de tratamiento termoquímico superficial, donde la superficie más externa de un material metálico es saturada con átomos de boro. El proceso de saturación de boro se da por medio de la difusión de átomos de este elemento a través de los espacios intersticiales de la red cristalina del metal (véase figura 6).

Durante el proceso, se desarrolla un crecimiento constante de la difusión (véase figura 7), que forma cristales tipo aguja, lo cual es característico para la difusión de boro, independientemente del tipo de técnica empleada. Una micrografía característica de este tipo de difusión se puede apreciar en la figura 8 .

Los materiales tratados con mayor frecuencia por el proceso de boronizado son el hierro y sus aleaciones. Para el caso de este tipo de materiales, la difusión de boro en la superficie viene acompañada de una reacción química, en la cual se forman fases cristalinas de boruros de hierro, como la $\mathrm{Fe}_{2} \mathrm{~B}$ y la $\mathrm{FeB}$ (véase figura 9), las cuales son las responsables de un gran número de propiedades [18]. Otros materiales, en los que también se ha reportado la aplicación del borizado, son las aleaciones de níquel, cobalto y titanio [19-21].

Existen diversas técnicas que permiten el boronizado de una pieza metálica. El proceso de termoboronizado es el más usado en el campo industrial, y consiste en promover la difusión del elemento boro, por medio del tratamiento a elevadas temperaturas. Esta técnica puede realizarse usando medios borizantes sólidos, líquidos o gaseosos.

El boronizado sólido requiere temperaturas entre 700 y $1.000{ }^{\circ} \mathrm{C}$, al igual que tiempos de tratamiento de hasta 10 horas (dependiendo del espesor de difusión requerido). En cuanto a la fuente de boro, esta varía en función de la técnica a emplear. Existen boronizados que se realizan con sustancias boronizantes en forma de polvos o en pastas. Generalmente dentro de su composición poseen carburo de boro, boro elemental o ferroboro como agentes boronizantes, borofluoruros de amonio, cloruro de sodio o cloruro de amonio, como agentes activadores de la reacción química, además de un medio inerte para la reacción, como alúmina, carburo de silicio o negro de humo [22].
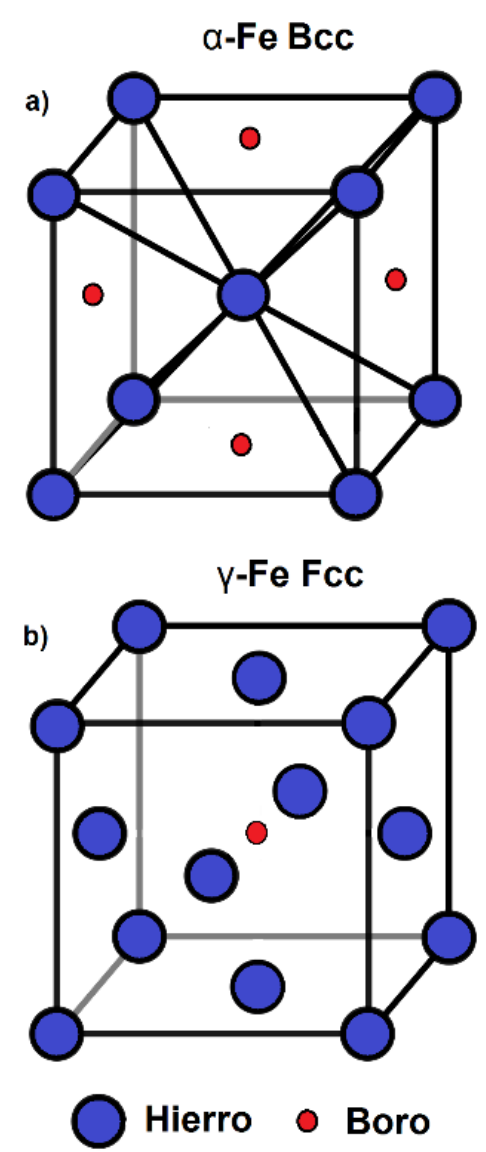

Figura 6. Celda unidad de (a) $\alpha$-Fe y (b) $\gamma$-Fe, con boro en espacios intersticiales. Fuente: elaboración propia.

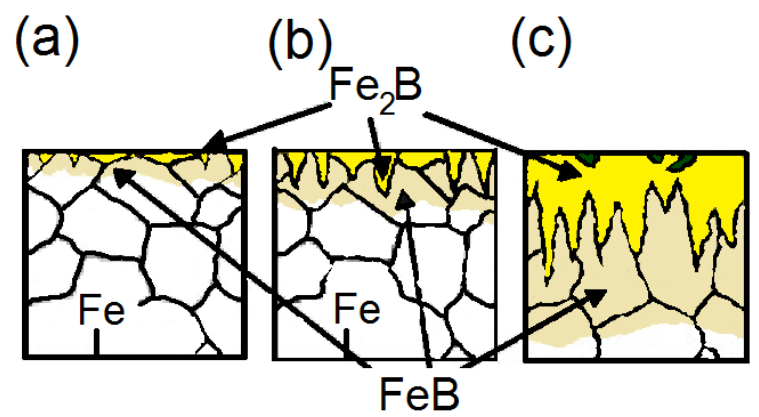

Figura 7. Patrón de crecimiento de la difusión de boro en tratamientos de boronizado. Fuente: Krukovich [16]. 


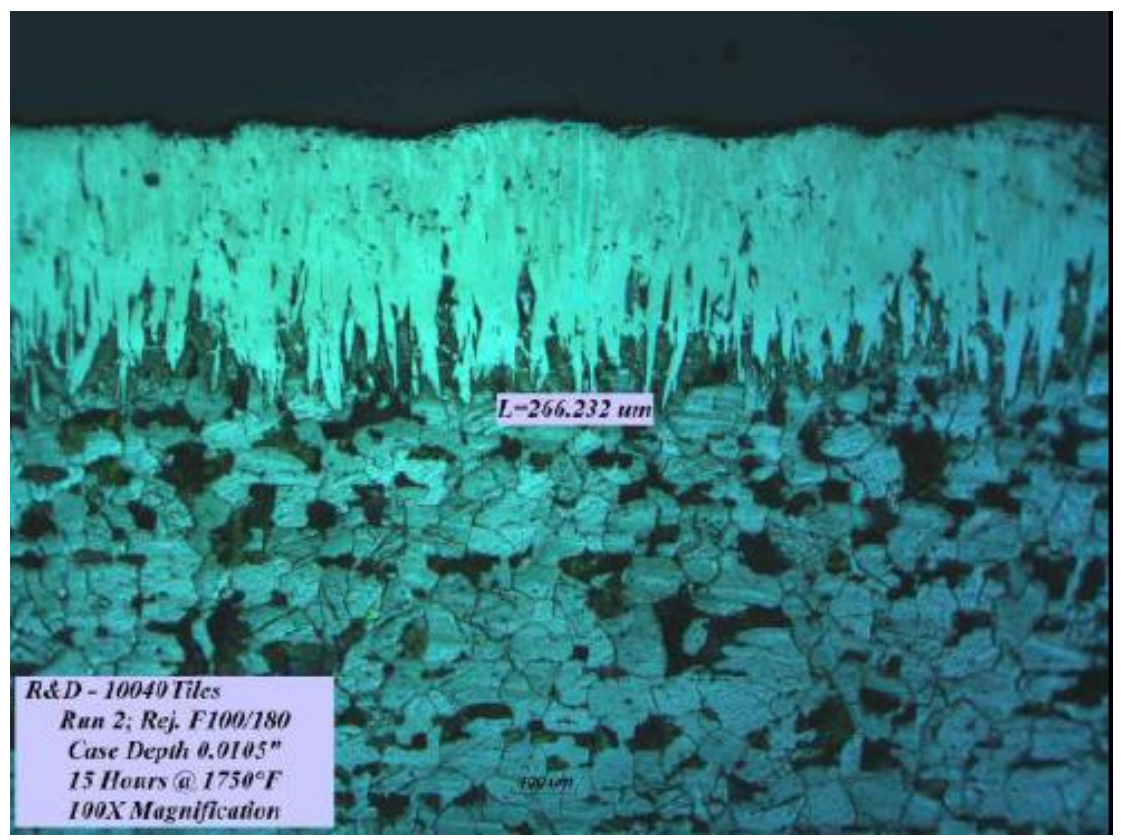

Figura 8. Micrografía de difusión térmica de boro. Fuente: Vadasz [17].
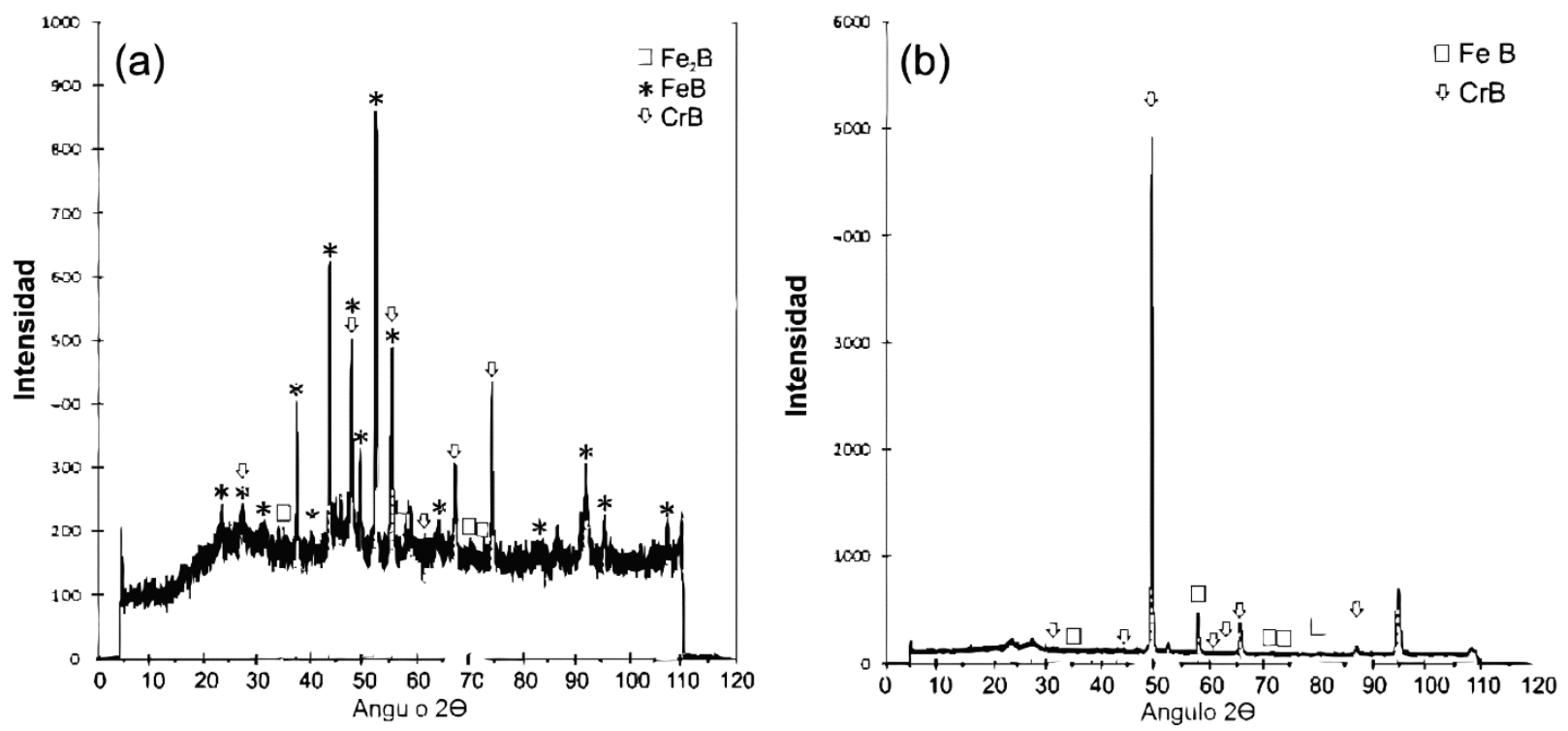

Figura 9. Patrón DRX de una difusión de boro sobre un acero AISI H13. Fuente: Cárdenas et al [18]. 


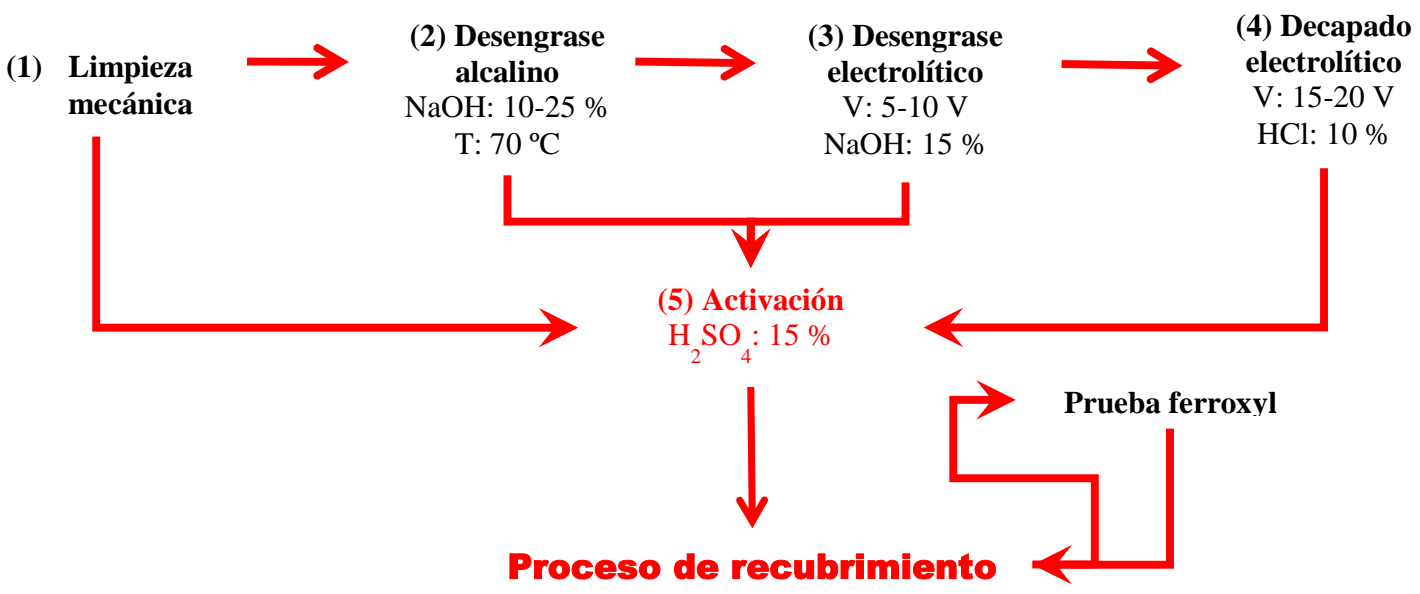

Figura 10. Esquema del proceso de tratamiento previo para las piezas a recubrir. Fuente: elaboración propia.

El boronizado líquido se puede realizar por medio de dos vías: electroquímica y no electroquímica. La diferencia entre ambas es la aplicación o no de una corriente eléctrica al medio para generar la reducción del boro. La vía no electroquímica se da por medio de una mezcla de borax con un agente reductor apropiado (carburo de boro, ferroaluminio, etc). Esta mezcla se calienta por encima de los $900{ }^{\circ} \mathrm{C}$ para obtener la fusión del medio borizante y generar un medio líquido. Para el caso del método electroquímico, el medio es similar al método anterior, con la diferencia de que el reactor se dispone de modo que se pueda tener una barra de grafito paralela a la pieza y que actúa como ánodo, mientras que la pieza a borizar opera como cátodo. Luego se aplica corriente continua a un determinado valor, mientras el cátodo gira sobre su eje para poder obtener una difusión uniforme [23,24].

El boronizado gaseoso es una técnica que usa precursores gaseosos de boro como agentes boronizantes en un medio reductor gaseoso, a temperaturas de 700 a $950{ }^{\circ} \mathrm{C}$. El precursor más común es del diborano mezclado con gas hidrógen. ${ }^{\circ}$ También se han reportado en la bibliografía otras formulaciones entre las que se incluyen compuestos organoborados como el trimetil borano y el trietil borano, los cuales son reducidos en atmósferas de nitrógeno/hidrógeno 75:25 [25,26].

Uno de los problemas más comunes que se presentan en piezas borizadas, el cual está asociado a las condiciones de trabajo de la pieza, es la aparición de microgrietas en la superficie debido: (i) al trabajo a temperaturas altas (de 250 a $450{ }^{\circ} \mathrm{C}$ ), (ii) a grandes presiones ejercidas sobre la superficie de la pieza (> $3 \mathrm{Ton} / \mathrm{m}^{2}$ ), (iii) a efectos cortantes cuando la pieza está sometida a torsión o (iv) la combinación de los procesos antes señalados actuando simultáneamente. Los casos más comunes de microgrietas están asociados a trabajos a altas temperaturas y las microgrietas surgen a consecuencia de la diferencia en el coeficiente de expansión térmica entre el sustrato y el compuesto intermetálico, debido a que el sustrato se expande más que el último a medida que aumenta la temperatura de trabajo. Si la temperatura es suficientemente alta $\left(>250^{\circ} \mathrm{C}\right)$ se fractura el compuesto intermetálico (boruro metálico) y la resistencia a la corrosión de la pieza queda comprometida, una vez que se generan las microgrietas, debido a la comunicación directa entre el medio ambiente y el sustrato.

En función a esta problemática que se presenta en el campo industrial (sobre todo en el sector petrolero), se planteó la necesidad de poder tener una alternativa para evitar el daño en piezas borizadas debido a este fenómen. ${ }^{\circ}$ Es por ello que se propone el uso del recubrimiento electroless nickel (Ni-P) como una técnica para reforzar la difusión de boro en piezas industriales borizadas, específicamente reforzamiento de tuberías de acero al carbono borizadas, las cuales son usadas ampliamente en el ámbito petrolero.

\section{Metodología}

Se emplearon probetas de acero al carbono grado API J55 , que se encontraban borizadas mediante el proceso EndurAlloyMR [17], realizado por Endurance Technologies Inc., Calgary, Canadá.

Para la presente investigación se comprobó el comportamiento del Ni-P sobre superficies borizadas, en términos de adherencia y difusión del recubrimiento en el compuesto intermetálico. Para ello se realizó un estudio preliminar, en el cual se sometió el sustrato a recubrir a una serie de procedimientos de limpieza previos, con el objeto de determinar cuál era la combinación ideal de pretratamientos para obtener una superficie uniformemente recubierta y libre de porosidad. En función de esto se diseñó el esquema mostrado en la 
figura 10. Se emplearon ocho probetas EndurAlloyMR para este paso de la investigación (dos por cada ruta de pretratamiento evaluada, véase tabla 1). Las probetas se trataron por pares según el pre-tratamiento a evaluar y posteriormente se recubrieron con una capa de Ni-P, para luego ser evaluadas empleando el procedimiento descrito en el apartado 7.4.2 de la norma ASTM B689, conocido como la prueba Ferroxyl, que permite detectar porosidad en el recubrimiento [27]. Esta prueba se aplica a sustratos ferrosos y consiste en la formación de un compuesto conocido como 'azul de Prusia', que tiene un color azul oscuro muy característico y que aparece por la reacción química entre el ferrocianuro de potasio (contenido en el reactivo Ferroxyl) y el hierro que pueda quedar expuesto en la superficie y que no fue recubierto por el Ni-P.

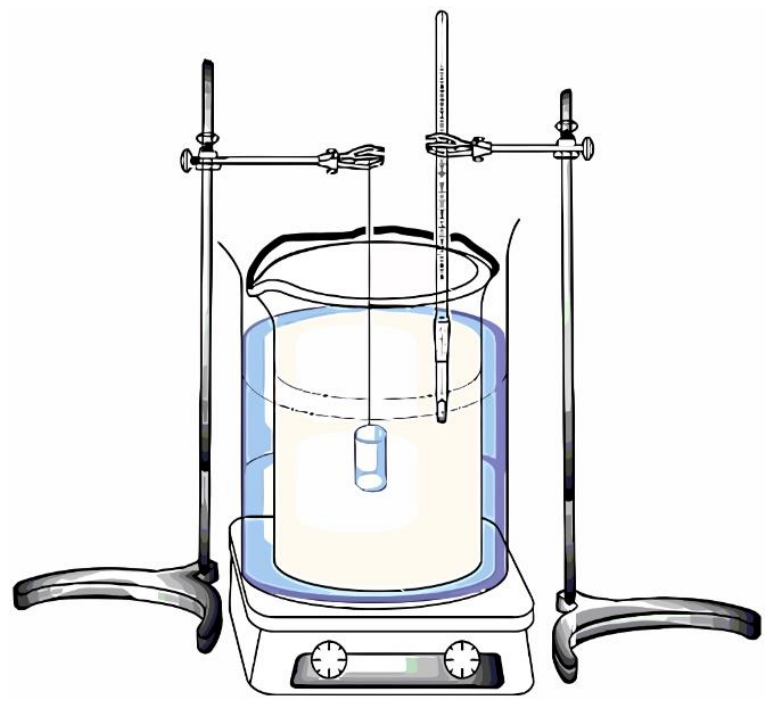

Figura 11. Montaje del baño de recubrimiento Ni-P. Fuente: elaboración propia.

Tabla 1. Rutas de pre-tratamiento y resultados de la prueba de ferroxyl.

\begin{tabular}{|c|c|c|}
\cline { 2 - 3 } \multicolumn{1}{c|}{} & Ruta tratamiento previo & $\begin{array}{c}\text { Prueba de } \\
\text { ferroxyl }\end{array}$ \\
\hline A & $(1) \rightarrow(5)$ & Positiva \\
\hline B & $(1) \rightarrow(2) \rightarrow(5)$ & Positiva \\
\hline C & $(1) \rightarrow(2) \rightarrow(3) \rightarrow(5)$ & Positiva \\
\hline D & $\begin{array}{l}(1) \rightarrow(2) \rightarrow(3) \rightarrow(4) \rightarrow \\
(5)\end{array}$ & Negativa \\
\hline
\end{tabular}

El procedimiento experimental empleado para el recubrimiento con $\mathrm{Ni}-\mathrm{P}$ de cada una de las probetas consistió en sumergirlas en un baño de recubrimiento ácido cuya composición se reporta en la tabla 2. Este baño fue ajustado a un $\mathrm{pH}=5$, una temperatura entre 75 y $80^{\circ} \mathrm{C}$, además de agitación magnética (véase figura 11).
Tabla 2. Composición química del baño de recubrimiento $\mathrm{Ni}-\mathrm{P}$.

\begin{tabular}{|c|c|c|c|}
\hline \multicolumn{4}{|c|}{ Constituyentes del baño } \\
\hline $\begin{array}{c}\text { Sulfato de } \\
\text { níquel (M) }\end{array}$ & $\begin{array}{c}\text { Hipofosfito de } \\
\text { sodio (M) }\end{array}$ & $\begin{array}{c}\text { Ácido } \\
\text { cítrico (M) }\end{array}$ & $\begin{array}{c}\mathrm{PbSO}_{4} \\
(\mathrm{M})\end{array}$ \\
\hline 0,2 & 0,5 & 0,21 & 0,001 \\
\hline
\end{tabular}

Una vez recubiertas y evaluadas las probetas por medio de la prueba ferroxyl, se eligió la ruta de pretratamiento que presentó los mejores resultados de ausencia de porosidad y se realizó a las muestras correspondientes un tratamiento térmico a una temperatura de entre 200 y 250 ${ }^{\circ} \mathrm{C}$ por una hora.

Posteriormente, las probetas se enfriaron empleando nitrógeno líquido y se cortaron transversalmente para evaluarlas por medio de microscopía electrónica de barrido, mediante un microscopio FEI-Quanta 250, acoplado a un analizador de energía dispersiva de rayos $\mathrm{X}(\mathrm{SEM} / \mathrm{EDX})$. Esta evaluación se hizo con el objeto de evaluar la morfología, así como también la distribución elemental, empleando las herramientas EDX-LineScan y EDX-Mapping.

\section{Resultados y discusión}

Todas las muestras recubiertas presentaron una superficie brillante posteriormente al proceso de recubrimiento. La tabla 1 muestra los resultados de las pruebas Ferroxyl para las probetas recubiertas mediante diferentes pretratamientos. Se puede apreciar que las probetas que no presentaron ningún tipo de porosidad fueron aquellas en donde se realizaron los cinco pasos de pretratamiento (ruta D). Esto indica que la superficie de este tipo de materiales requiere de una limpieza exhaustiva para lograr un buen recubrimiento $\mathrm{Ni}-\mathrm{P}$ ya que la difusión de boro, a pesar de que forma un compuesto intermetálico sumamente denso, típicamente presenta una superficie altamente porosa y esa porosidad puede alojar distintos tipos de contaminación que pueden afectar el desarrollo del recubrimiento durante el proceso y generar zonas porosas que afectan su resistencia a la corrosión.

La figura 12 corresponde a la micrografía del corte transversal de la probeta D. En la micrografía se puede apreciar la morfología de 'tipo coliflor'. Este tipo de morfología es característica de los recubrimientos Ni-P y ha sido ampliamente reportada por diversos autores [13$15]$. 


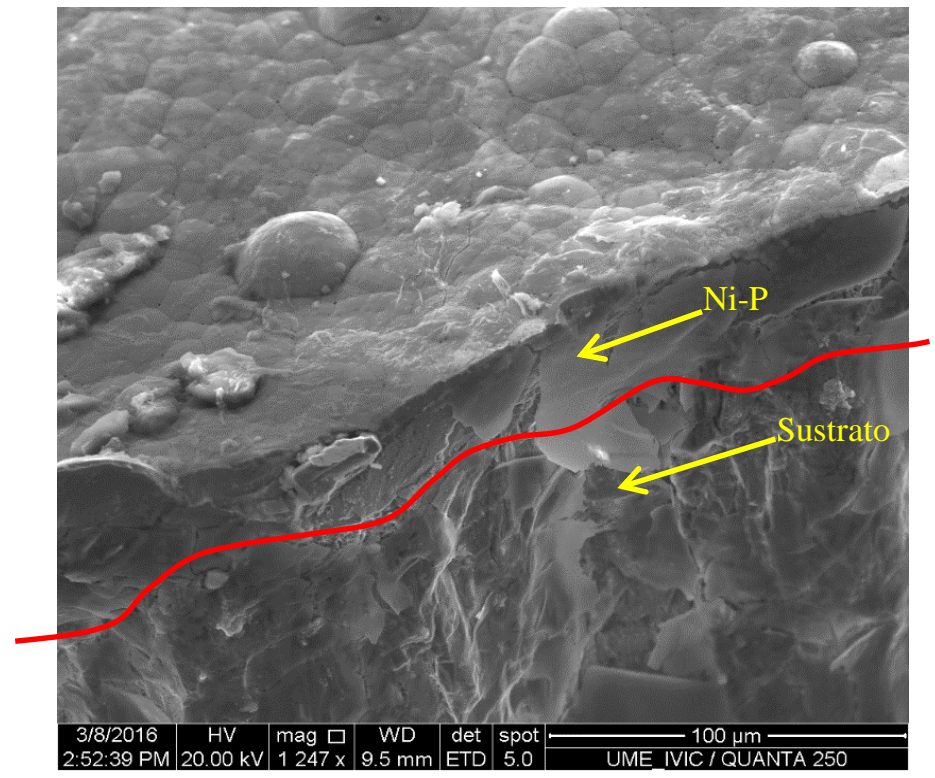

Figura 12. Micrografía del corte transversal de la probeta recubierta con Ni-P. Fuente: elaboración propia.

(a)

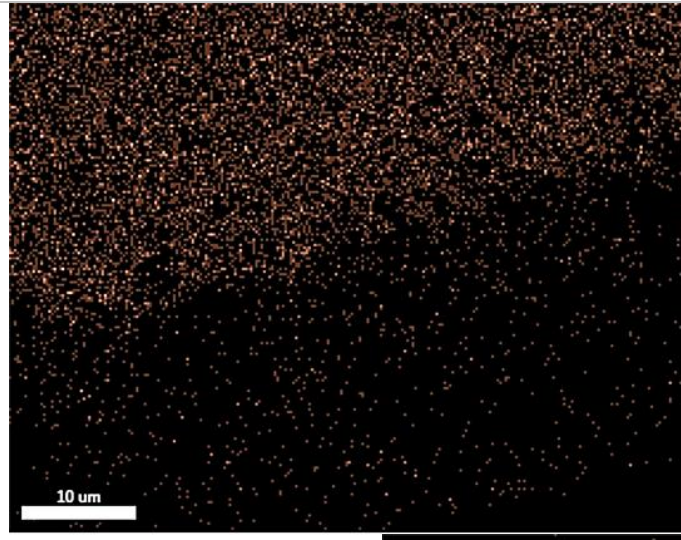

(b)

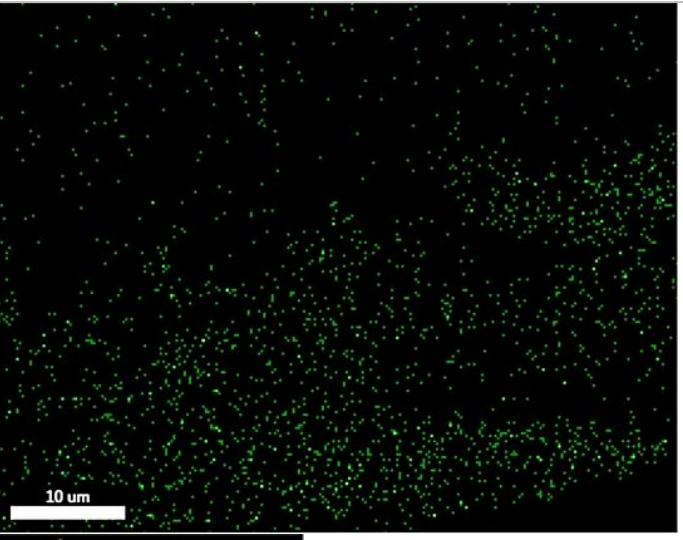

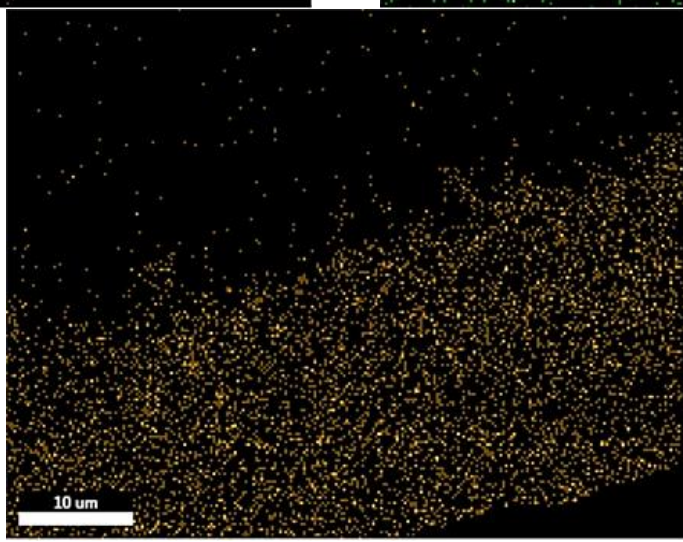

(c)

Figura 13. Micrografías de los análisis por EDX-Mapping para (a) hierro; (b) fósforo y (c) níquel. Fuente: elaboración propia. 
Uno de los aspectos más importantes planteado en la presente investigación es la distribución de los elementos químicos a lo largo de la sección transversal de recubrimiento y en la zona entre la cobertura Ni-P y la difusión de boro, esto a objeto de determinar si existió o no difusión de un material en el otro. Las figuras 13, 14 y 15 muestran ampliamente y en detalle cómo ocurrió la distribución de los elementos entre ambos materiales. Las micrografías presentadas en la figura 13 muestran cómo es la distribución de los elementos constituyentes de la muestra y que pueden ser detectados por la técnica EDX: hierro, níquel y fósforo.

Cada punto en las micrografías representa una zona analizada con el detector EDX-MEB. La micrografía 13(a) corresponde a la distribución del elemento hierro, el cual es el constituyente principal del sustrato, que se puede observar por la mayor densidad de puntos naranja en la parte superior de la micrografía, y que disminuye significativamente en la medida en que va del sustrato al recubrimiento. La micrografía 13(b) corresponde a la distribución del elemento fósforo en la muestra, que el cual presenta una mayor concentración en la zona baja de la imagen correspondiente al recubrimiento. En la micrografía 13(c) se aprecia la distribución del elemento níquel en la zona del recubrimiento. Uno de los aspectos más destacables de los resultados obtenidos en el análisis de EDX-Mapping es la detección de los elementos níquel y fósforo en la zona de difusión de boro, así como también el elemento hierro en la zona del recubrimiento. Esto permite afirmar que existe una difusión del recubrimiento Ni-P en la superficie del sustrato.

Esta afirmación se corrobora con las figuras 14 y 15, que corresponden al análisis EDX-LineScan, realizado para la misma zona de trabajo que para EDX-Mapping.

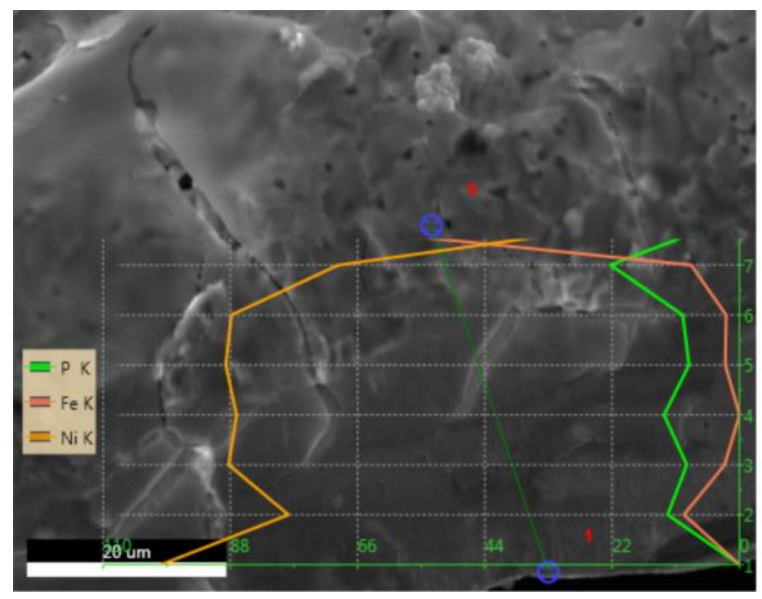

Figura 14. Micrografía de la zona de análisis EDXLineScan. Fuente: elaboración propia.

La figura 14 muestra una micrografía de la zona analizada, y sobre esta se encuentra superpuesta una línea color verde, que se usa para marcar el camino por el cual el detector EDX realizo el análisis. En este caso se eligió un barrido a lo largo de las primeras $40 \mu \mathrm{m}$ desde la superficie de la pieza y a través del corte transversal hasta llegar a la zona del sustrato. El análisis registró las concentraciones relativas de los elementos níquel, fósforo y hierro a lo largo del recorrido.

La figura 15 muestra un gráfico del porcentaje relativo en peso del elemento con respecto a la distancia en $\mu \mathrm{m}$ desde la superficie del recubrimiento hasta el sustrato. En la misma figura se observa la presencia significativa de una pequeña cantidad de hierro a lo largo del recubrimiento hasta llegar a los $35 \mu \mathrm{m}$ de profundidad. Una vez se ha llegado a este punto, ocurre un cambio significativo en las concentraciones de los elementos a lo largo de una franja de aproximadamente $5 \mu \mathrm{m}$, en el cual las

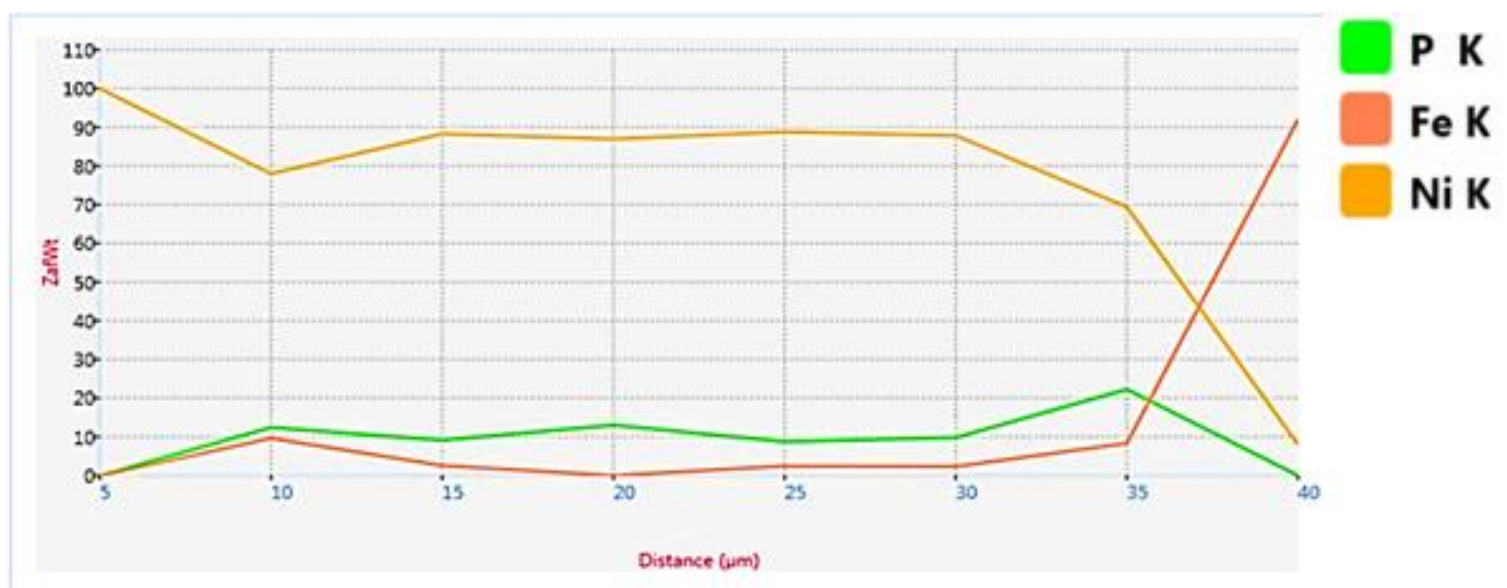

Figura 15. Gráfico de distribución elemental en la zona frontera entre el recubrimiento Ni-P y la difusión de boro. Fuente: elaboración propia. 
concentraciones de níquel y fósforo caen progresivamente mientras que la de hierro aumenta hasta llegar al $100 \%$.

Estos resultados no solo corroboran la difusión del Ni-P observada en el sustrato, sino que, adicionalmente, indica que existe una zona "interfacial", que en el área de análisis es de aproximadamente $5 \mu \mathrm{m}$, en donde hay una fuerte interacción entre los dos materiales.

El hecho de que exista una difusión de esta magnitud en el sustrato plantea un reto importante en pro de determinar en qué parte del proceso descrito para generar el recubrimiento Ni-P ocurre la difusión de este en la capa, para diferenciar, por ejemplo, si el tratamiento térmico influye en la difusión, y, de ser así, determinar hasta donde es posible optimizar el mismo tratamiento para obtener una mayor penetración en el sustrato estudiado.

\section{Conclusiones}

El proceso de recubrimiento de níquel fosforado se encuentra fuertemente influenciado por el estado inicial del sustrato a recubrir. Para el caso de sustratos de acero con difusión de boro, se hace necesario un extenso procedimiento de limpieza fisicoquímica que permita eliminar impurezas, óxido y partículas contaminantes que puedan influir en la generación de un sustrato poroso. La capa de níquel fosforado mostró un excelente cubrimiento, posterior a un proceso de pre-tratamiento del sustrato en el que se incluyó una limpieza mecánica, un desengrase alcalino, un desengrase y decapado electrolíticos y una activación ácida.

En cuanto a la interacción del recubrimiento con el sustrato, se pudo comprobar que existe una difusión del níquel fosforado en la difusión de boro, lo cual le da al recubrimiento una excelente adherencia y aumenta significativamente la probabilidad de cubrir las microgrietas que pueda poseer la difusión de boro en las piezas de acero borizado.

\section{Referencias}

[1] R. C. Agarwala y V. Agarwala, "Electroless alloy/composite coatings: A review," Sadhana, vol. 28, núm. 3, pp. 475-493, 2003. doi: 10.1007/BF02706445

[2] A. Brenner y G.E. Riddell, "Nickel Plating on Steel by Chemical Reduction," Journal of Research of the National Bureau of Standards, vol. 37, pp. 31-34, 1946.

[3] H.J. West, "Electroless Nickel Barrel Plating," Metal Finishing, vol. 52, no. 3, pp. 64, 1954.
[4] H.J. West, "Electroless Nickel Plating on Nonferrous Metals," Metal Finishing, vol. 52, no.7, pp. 72, 1954.

[5] A.H. Graham, R.W. Lindsay y H.J. Read,"The Structure and Mechanical Properties of Electroless Nickel," Journal of the Electrochemical Society, vol. 112, no. 4, pp. 401-413, 1965.

[6] K.S. Rajam, I. Rajagopal, S.R. Rajagopalan y B. Viswanathan. "DSC, X-ray and magnetic studies on electroless Ni-P films grown in alkaline ethanolamine baths," Materials Chemistry and Physics, vol. 33, pp. 289-297, 1993.

[7] U.I. Apachitei y J. Duszczyk, “Autocatalytic nickel coatings on aluminium with improved abrasive wear resistance," Surface and Coatings Technology, vol. 132, pp. 89-98, 2000.

[8] S.B. Antonelli, T.L. Allen, D.C. Johnson y V.M. Dubin, "Crystallization Behavior of Ni-P Alloy Films on Co and Cu Seed Layers," Journal of The Electrochemical Society, vol. 152, no. 9, pp. J120-J124, 2005.

[9] R.C. Agarwala, S.Z. Ray, "Variation of structure in electroless Ni-P films with phosphorous content," Zeitschrift fuer Metallkunde/Materials Research and Advanced Techniques, vol. 79, no. 7, pp. 472-475, 1988.

[10] K. G. Keong, W. Sha, y S. Malinov, "Crystallisation kinetics and phase transformation behaviour of electroless nickel-phosphorus deposits with high phosphorus content," J. Alloys Compd., vol. 334, no. 1, pp. 192-199, 2002. doi: 10.1016/S0925-8388(01)017984

[11] J. N. Balaraju, S. M. Jahan, A. Jain, y K. S. Rajam, "Structure and phase transformation behavior of electroless Ni-P alloys containing tin and tungsten," $J$. Alloys Compd., vol. 436, no. 1-2, pp. 319-327, 2007. doi: 10.1016/j.jallcom.2006.07.045

[12] J.R. Henry, "Electroless (autocatalytic) plating," Metal Finishing, vol. 97, no. 1, pp. 431-442, 1999. doi: 10.1016/S0026-0576(01)85302-2

[13] X. Fan, L. Gu, S. Zeng, L. Zhu, C. Wang, Y. Wang y X. Cao, "Improving stability of thermal barrier coatings on magnesium alloy with electroless plated $\mathrm{Ni}-\mathrm{P}$ interlayer," Surf. Coatings Technol., vol. 206, no. 21, pp. 4471-4480, 2012. doi: 10.1016/j.surfcoat.2012.05.004

[14] D. Seifzadeh, K. Mohsenabadi, Z. Rajabalizadeh, "Electroless Ni-P plating on magnesium alloy by innovative, simple and non-toxic oxalate pretreatment 
Caracterización por microscopía electrónica de barrido del recubrimiento no electrolítico de níquel (electroless nickel)

and its corrosion protection," RSC Advances, vol. 6, no. 99, pp. $97241-97252$, $2016 . \quad$ doi: 10.1039/C6RA19984D

[15] I. Baskaran, S. Narayanan y A. Stephen, "Effect of accelerators and stabilizers on the formation and characteristics of electroless Ni-P deposits," Materials chemistry and physics, vol. 99, no. 1, pp. 117-126, 2006. doi: 10.1016/j.matchemphys.2005.10.001

[16] M. G. Krukovich, B. A. Prusakov y I. G. Sizov, Plasticity of boronized layers. Switzerland: Springer International, 2016.

[17] A. Vadasz. "Technical considerations for implementing enduralloy hardened production tubing in an oil producing area," in Panamerican Mature Fields Congress, Veracruz, Mexico, 2015, pp. 15- 159.

[18] E. E. Cárdenas et al. "Characterization and wear performance of boride phases over tool steel substrates", Adv. Mech. Eng., vol. 8, no. 2, 2016. doi: $10.1177 / 1687814016630257$

[19] K. G. Anthymidis et al. "Boriding of nickel in a fluidized bed reactor," Materials Research Bulletin, vol. 37, no. 3, pp. 515-522, 2002. doi: 10.1016/S00255408(02)00672-4

[20] G. L. Zhunkovskii, B. S. Navrotskii, y Y. P. Kolosvetov, "Boronizing of cobalt and some cobalt base alloys," Sov. Powder Metall. Met. Ceram., vol. 11, no. 11, pp. 888-890, 1972. doi: 10.1007/BF00806648

[21] K. G. Anthymidis, D. N. Tsipas y E. Stergioudis. "Boriding of titanium alloys in a fluidized bed reactor," Journal of materials science letters, vol. 20, no. 22, pp. 2067-2069, 2001.

[22] D. N. Tsipas y J. Rus, "Boronizing of alloy steels," J. Mater. Sci. Lett., vol. 6, no. 1, pp. 118-120, 1987. doi: 10.1007/BF01729451

[23] T. W. Spence y M. M. Makhlouf, "Characterization of the operative mechanism in potassium fluoborate activated pack boriding of steels," Journal of materials processing technology, vol. 168, no. 1, pp. 127-136, 2005. doi: 10.1016/j.jmatprotec.2004.10.015

[24] P. A. Dearnley y T. Bell, "Engineering the surface with boron based materials," Surface Engineering, vol. 1, no. 3, pp. 203-217, 1985. doi: 10.1179/sur.1985.1.3.203

[25] A. Küper, X. Qiao, H. R. Stock y P. Mayr, "A novel approach to gas boronizing," Surface and Coatings
Technology, vol. 130, no. 1, pp. 87-94, 2000. doi: 10.1016/S0257-8972(00)00682-4

[26] N. Makuch, M. Kulka y M. Popławski, “Two-stage gas boriding of Nisil in $\mathrm{N}_{2}-\mathrm{H}_{2}-\mathrm{BCl}_{3}$ atmosphere," Surface and Coatings Technology, vol. 244, pp. 78-86, 2014. doi: 10.1016/j.surfcoat.2014.01.057

[27] Standard Specification for Electroplated Engineering Nickel Coatings, ASTM B689, 2003. 